\title{
Análisis de las noticias sobre lectura publicadas en el periódico El País (2004-2010)
}

Analysis of the news on reading published in El País (2004-201)

\author{
Belen García-Delgado Giménez \\ Universidad Europea de Madrid, Urb. El Bosque C/ Tajo s/n, \\ 28670 Villaviciosa de Odón (Madrid), belen.garcia-delgado@uem.es
}

\begin{abstract}
Resumen
Análisis de los discursos públicos sobre lectura incluidos en las noticias del periódico El País. Se estudiaron las frecuencias de los términos más relevantes para el tema objeto de estudio, con especial atención a las secciones en las que se publicaron, su relación con los cambios de gobierno, comparándolos con otros periódicos de gran tirada, como El Mundo y $A B C$.
\end{abstract}

Palabras clave: Lectura. Noticias. Sociología de la lectura. Hábito lector. Lectura y comunicación.

\section{Introducción}

El principal motivo por el que se realiza este trabajo es el interés que despierta a nivel cultural la lectura. Esta ha sido y sigue siendo el paradigma de la cultura en la sociedad. A pesar de los múltiples soportes en los que se puede presentar en la actualidad - papel, digital, CDROM, etc.-, en todos se debe llevar a cabo un proceso de descodificación de la información. Bien es cierto que hay otras formas de adquirir conocimientos, como por ejemplo mediante un proceso pasivo de desciframiento de la información -a través de la escucha, el visionado, etc.- pero, en todo caso, se precisa de la realización de una lectura previa por parte del interlocutor. Es más, todo objeto cultural además de basarse en la lectura, incita a la misma.

A pesar de su relevancia, resulta muy difícil estudiar de manera racional una realidad tan íntima, personal e intangible como es la lectura (Lahire, 2004). En opinión de Lahire, al analizarla, se está destruyendo la relación mágica que existe entre las obras y sus lectores, ya que se está manejando como cualquier otro objeto de estudio. Se trata de calcular algo que es muy difícil de cuantificar, ya que es algo inmaterial que forma parte de la vida privada de cada persona. De esta forma, el enfoque estadístico de la lectura lo único que consigue es poner en evidencia tendencias generales, pero no permite

\begin{abstract}
Analysis of the public-officials' discourses on reading published in the daily newspaper El País. This project is based on a frequency study where the most relevant terms have been analyzed to observe the evolution of public discourse throughout the period of time 2004 to 2010, paying special attention to the sections in which news appear and their relation with government changes, and comparing the results with other relevant Spanish newspapers, as El Mundo y $A B C$.
\end{abstract}

Keywords: Reading. News. Reading sociology. Reading habits. Reading and communication.

una estimación ni una construcción exhaustiva de los distintos tipos de lectores (Bahloul, 2002).

Debido a que la prensa es uno de los medios que se está viendo más afectado por los distintos cambios que tienen lugar en la sociedad, este artículo se centra en dicho medio. Se trata de un análisis más objetivo que el de los discursos privados, aunque también presenta dificultades. Por este motivo, se seleccionarán términos relevantes para el concepto de la lectura, atendiendo al valor semántico de los mismos. Este proceso se explicará en detalle en el apartado siguiente.

\section{Objetivos}

El motivo fundamental por el que se han abordado los discursos públicos sobre lectura, es que el proceso de análisis es más sistemático que en el caso de los discursos privados. Sin duda, en los discursos públicos resulta más fácil determinar una serie de indicadores a seguir, para así estudiar los mismos factores de forma coherente y equitativa en los distintos tipos de medios.

De esta forma el objetivo de este trabajo es analizar el valor que ha ido adquiriendo la lectura en uno de los medios de comunicación social de mayor relevancia en España -en concreto, El País- en este periodo cronológico objeto de estudio. En este sentido se tendrá en cuenta la percepción que se tiene de la misma, es decir, 
si la lectura se considera un proceso cultural, tecnológico, político, social, etc. Posteriormente, se estudiará la frecuencia y contenido de los términos que tienen especial relevancia para el mundo de la lectura en los medios de comunicación social. De este modo se analizará la frecuencia que tienen los términos: 'libro', 'lectura', 'lectores', 'cultura', 'biblioteca', 'televisión', 'educación', 'hábitos', 'universidad', 'universitarios', 'internet' 'comunicación', así como el valor semántico de los mismos, es decir qué palabras se encuentran antes y después de estos términos objeto de estudio. Se trata de una forma de averiguar por ejemplo, en qué formato se lee en papel o en digital-, qué género, etc.

Por tanto, el rango de fechas tenido en cuenta para la selección de noticias ha sido desde el uno de enero de 2004 hasta el treinta y uno de diciembre de 2010. Para la acotación de este período se han tenido en cuenta aspectos como el tipo de gobierno que se encontraba en el poder (PSOE), que supone un cambio con respecto al rango temporal inmediatamente anterior, así como la relevancia que tiene debido al despegue de las nuevas tecnologías que tuvo lugar durante el mismo.

\section{Metodología}

La herramienta que ha servido para realizar el seguimiento ha sido Factiva, una base de datos de prensa que contiene noticias de agencias y también de más de diez mil periódicos de ámbito nacional, internacional y local. Este recurso exige suscripción y da acceso al contenido de noticias de un amplio rango de fechas, aunque la antigüedad de las mismas es variable según el periódico del que se trate. En todos los casos las publicaciones incluidas cuentan con al menos diez años de antigüedad.

En cuanto a la descripción bibliográfica, clasificación y organización de textos, se ha manejado el gestor de contenidos RefWorks. Dicha herramienta presenta grandes ventajas para el estudio y análisis del contenido de los textos, aunque nutrirla de textos resultó ser una ardua tarea.

Además, se han utilizado dos aplicaciones para el análisis terminológico. Wordsmith Tools nos ha permitido conocer la frecuencia de aparición de los textos (absoluta y por grupos de textos) de palabras relacionadas con el concepto de lectura - todos ellos recogidos en la tabla I- , para posteriormente establecer las distintas comparaciones tanto por épocas como por las fuentes. La aplicación Tropes ha permitido realizar un análisis de la carga semántica de las palabras. Este programa proporciona unos grá- ficos de gran interés que facilitan la interpretación de los datos al establecer las relaciones entre los términos teniendo en cuenta sus categoría morfológicas — verbos, adverbios, adjetivos...- de forma que si, por ejemplo en el corpus de noticias se nombra en diversas ocasiones a diferentes autores literarios, en lugar de analizar cada palabra como ente aislado Follet, Zafón...- se analizan todas ellas en conjunto como 'nombres propios'. Muestra de ello son los Gráficos I y II incluidos en el apéndice. En el Gráfico I los términos más representativos de los textos aparecen representados por esferas, el tamaño de las mismas es proporcional al número de palabras contenidas en el corpus de textos, asimismo las esferas de color azul son las que se encuentran antes del concepto clave de nuestro estudio - lectura - y las verdes las que están después de la misma. Otra modalidad de gráfico que nos ofrece este programa es el Gráfico II, donde esta vez los términos de color azul son los que encuentran antes de la palabra 'lectura' en los distintos textos, mientras que los rosas se posicionan después de la misma.

\begin{tabular}{lrrrr}
\hline Términos & 1977 & 1982 & 1996 & 2004 \\
& a 1982 & a 1996 & a 2004 & a 2010 \\
\hline Libro & 86 & 88 & 68 & 73 \\
\hline Lectura & 66,6 & 86 & 82 & 88 \\
\hline Cultura & 26,6 & 37 & 68 & 57 \\
\hline Biblioteca & 73 & 51 & 65 & 61 \\
\hline Televisión & 40 & 21 & 25 & 15 \\
\hline Educación & 13 & 23 & 25 & 34 \\
\hline Hábitos & 26,6 & 27 & 20 & 15 \\
\hline Comunicación & 20 & 17 & 57 & 53 \\
\hline Universidad & 13,3 & 13 & 5,7 & 7,6 \\
\hline Universitarios & - & 7,8 & 17 & 7,6 \\
\hline Internet & - & - & 22 & 34 \\
\hline
\end{tabular}

Tabla I. Índice de frecuencias del periódico El País por períodos históricos

\section{Resultados generales}

Se recogieron un total de 26 noticias sobre lectura en el periódico El País durante este período temporal. Pero, aparte de comentar estos resultados, se señalarán también los cambios significativos que han tenido lugar con respecto a períodos anteriores. Esto se debe a que estamos ante un artículo que consta de tan solo una parte de un proyecto más amplio en el que se 
analizan los discursos legislativos y medios de comunicación social -El País, El Mundo y
ABC- entre 1960 y 2010 (García-Delgado, 2012).

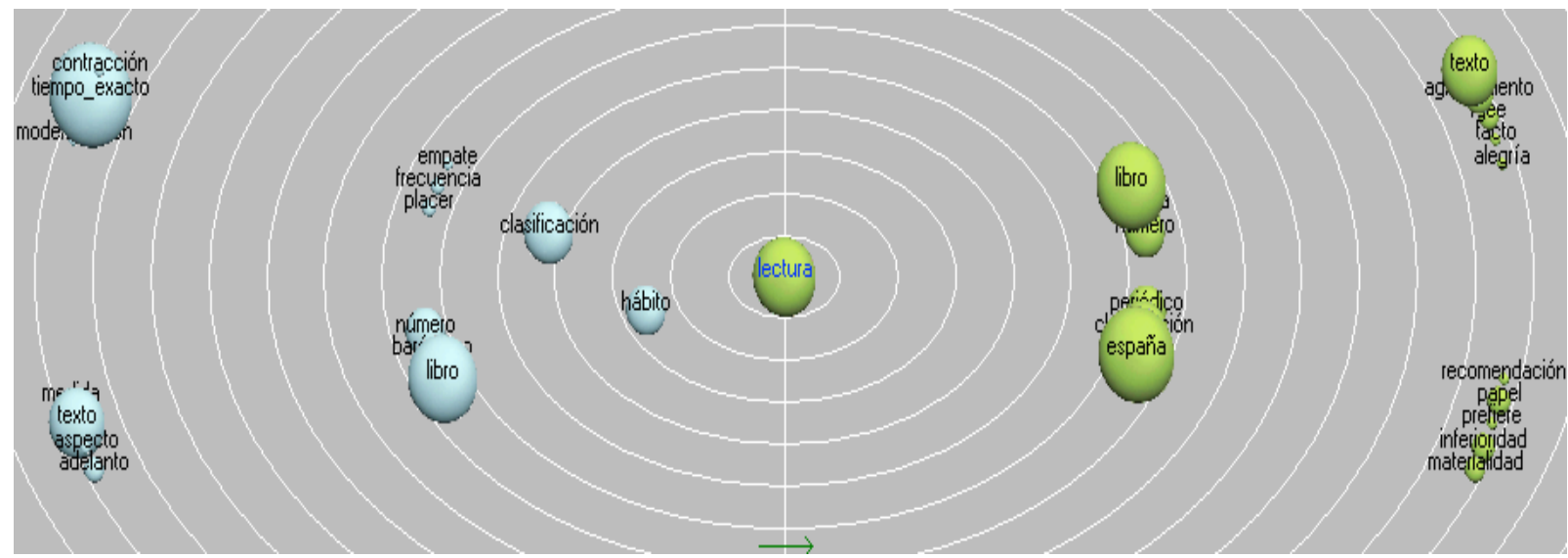

Gráfico I. El término 'lectura’ en El País de 2004 a 2010

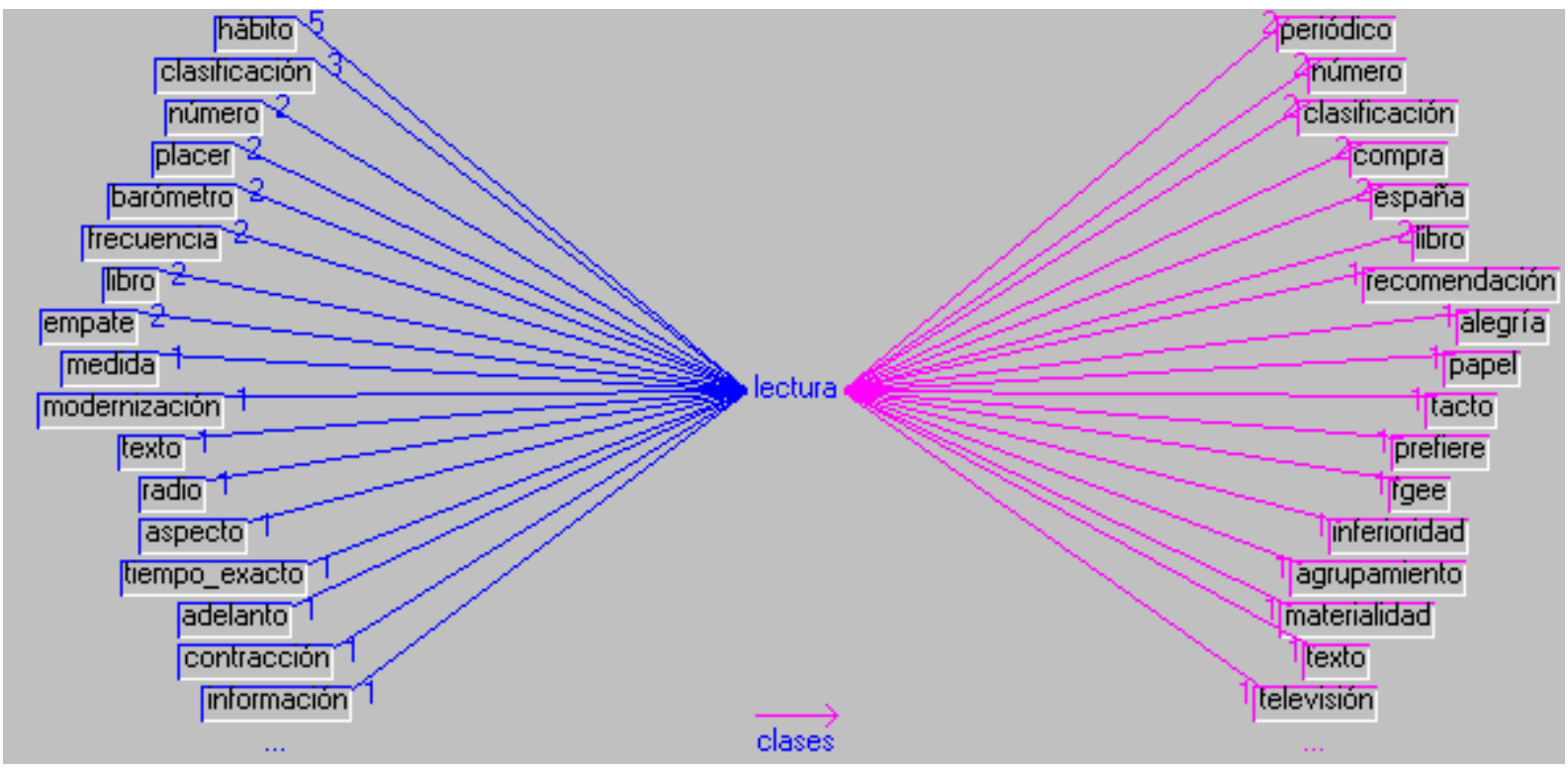

Gráfico II. El término 'lectura’ en El País de 2004 a 2010

Observando la Tabla I y cotejando los resultados obtenidos con respecto a períodos temporales anteriores podemos afirmar lo siguiente. El término 'biblioteca', ha bajado con respecto a los demás períodos, por lo que parece que no se le da tanta importancia como en el período comprendido entre 1977 y 1982. De hecho apenas encontramos noticias que versen sobre este tipo de centros, como, por ejemplo, Las bibliotecas son los equipamientos culturales más utilizados en la provincia de Barcelona del 24/11/2005. Lo mismo ocurre con 'educación', que ha bajado bastante con respecto a los anteriores rangos temporales, lo cierto es que estas son muy escasas, como, por ejemplo, La lectura se hace cuesta arriba: Maestros, padres y edito- res constatan el desapego al libro entre los alumnos del 02/03/2010.

'Cultura' sigue siendo frecuente, aunque ha bajado ligeramente con respecto al período anterior (1996 a 2004), pero no deja de ser mayoritario, por lo que la lectura sigue considerándose una actividad eminentemente cultural.

Por otro lado, 'lectores' sigue siendo frecuente, probablemente por los diversos estudios sobre hábitos de lectura, aunque -eso sí- ya no con tanta fuerza como en el período anterior. Probablemente se deba a que al no ser en ese momento tan novedoso -ya que el Barómetro de hábitos de lectura y compra de libros lleva publicándose desde el año 2000-, no se le da tanta importancia como antes. Sin embargo se sigue 
hablando de 'hábitos' —con una frecuencia del $53 \%$ - de lectura. 'Comunicación' ha subido 10 puntos con relación al período anterior; pero, sin embargo, no llega a alcanzar la frecuencia del primer período, algo que no deja de ser sorprendente, ya que este período temporal se caracteriza por el desarrollo de las nuevas tecnologías: Internet, dispositivos lectores, etc. Esto se debe al uso de palabras que sustituyen este término, aunque se refieren al mismo concepto, como ocurre con 'medios' con un $11 \%$ de frecuencia.

'Universidad' y 'universitarios' siguen teniendo una frecuencia baja, llegando a descender 10 puntos con respecto al período anterior. Pero, al igual que ocurría con el término anterior, estos se sustituyen por otros como 'estudiantes' con un $8 \%$, 'alumnos' con un $4 \%$ y 'joven' con un $8 \%$.

Por otro lado, 'internet' ha subido con respecto al período anterior, pero sigue siendo bajo para lo que realmente ha supuesto en la sociedad actual. Esto se debe a que se han utilizado otras palabras en su lugar como 'red' con un 19\%, 'web' con un $7 \%$ y 'pantalla' con un $12 \%$. Comprobamos de esta manera que son diversas las palabras que se emplean para hacer alusión al mismo concepto, Internet.

En el Gráfico I encontramos ciertas similitudes y algunas diferencias con respecto al período temporal anterior. Empecemos por la más evidente, que es la mayor distancia del término 'hábito' con respecto a 'lectura': El 58\% de los gallegos no ha leído un libro en el último año del 08/11/2007, El 60\% de los vascos dice que lee todos los días del 16/02/2005, Euskadi lee más libros que el resto de España del 25/11/2008, La frecuencia de lectura y el número de lectores crecieron durante 2004 del 07/02/2005. Ahora además encontramos textos relacionados con las características de consumo cultural de los españoles: La radiografía del consumidor cultural del 28/11/2007, Los españoles prefieren el cine y la música entre las opciones culturales del 01/04/2005 y Cultura por debajo de la media del 13/04/2005. Observamos también de nuevo 'tiempo exacto' y 'frecuencia' en el Gráfico I, ambos referidos a la cantidad de tiempo empleada en leer, períodos en los que se lee, etc.: El 58\% de los gallegos no ha leído un libro en el último año del 08/11/2007 y El 60\% de los vascos dice que lee todos los días del 16/02/2005.

La razón principal por la que encontramos gran número de noticias sobre los hábitos de lectura y consumo cultural es que una de las principales encuestas realizadas en España sobre este tema - Barómetro de hábitos de lectura y com- pra de libros- fue publicada por primera vez en el año 2000. A partir de la misma surgieron similares estudios en los años posteriores.

Por este motivo términos como 'clasificación', está presente, refiriéndose a los géneros de lectura preferidos por los lectores: Los españoles compran más prensa hoy que hace cinco años del 19/08/2007, El grueso de lectores de revistas culturales son hombres del 06/10/2007, Enganchados al 'thriller' histórico: La mitad de los 30 libros más leídos en 2006 en España sigue la senda de 'El código Da Vinci' del $22 / 2 / 2007$ y El futuro de la poesía no cabe en los libros del 12/08/2009.

Esta última noticia se relaciona además con el concepto 'recomendación', pues diferentes autores sugieren la lectura y compra de ciertos títulos, 'texto[s]' tal y como vemos en el Gráfico I.

Por otro lado, encontramos el término 'periódico', destacando la importancia que se le da a la lectura de diarios, probablemente debido al éxito que tiene en esta época la prensa electrónica: Prensa. Francia. 'Le Monde' reduce tamaño, aumenta el tipo de letra y da más fotografías del $7 / 11 / 2005$. Pero, esta vez 'libro' aparece en una esfera de tamaño mayor que en el rango temporal anterior, por lo que la frecuencia ha aumentado. Esto se debe al auge de las nuevas tecnologías, obligando a diferenciar las preferencias entre los distintos géneros y formatos de lectura: La mitad de los españoles no sabe qué es un libro electrónico del 16/07/2009. Vemos además la referencia que se hace a los nuevos formatos en el Gráfico I, ya que aparece el término 'papel', refiriéndo implícitamente a los nuevos soportes de lectura.

Todos estos datos aparecen recogidos mayoritariamente en el Barómetro de hábitos de lectura y compra de libros, por eso encontramos el término 'barómetro' de nuevo en el gráfico. De ahí que vuelva a aparecer 'número', referente a la cantidad de libros leídos, presente en noticias como Euskadi lee más libros que el resto de España del 25/11/2008 y La frecuencia de lectura y el número de lectores crecieron durante 2004 del 07/02/2005.

Algo que ha cambiado en esta época es 'placer' por la lectura, inexistente en los anteriores períodos, al igual que 'alegría'. Por este motivo, parece que cada vez se reflexiona más sobre los beneficios de la lectura.

Otra novedad es la referencia que se hace a las bibliotecas, presentes en el gráfico bajo la palabra 'agrupamiento', tal y como se observa en las noticias que hacen alusión a la misma: Las bibliotecas son los equipamientos culturales más 
utilizados en la provincia de Barcelona del $24 / 11 / 2005$, aunque no muy abundantes, tal y como se expuso en líneas anteriores.

En cuanto a las relaciones de palabras que ofrece este programa Tropes, vemos que aparece por primera vez - ya que no aparecía en los anteriores períodos- el libro relacionado con la forma, es decir se hace alusión a los nuevos formatos, al libro electrónico; pero su frecuencia es aún muy baja.

\section{Secciones que ocupan las noticias acerca de lectura en el periódico El País de 2004 a 2010}

En este apartado se analiza la percepción que se tiene de la lectura, es decir, si la lectura se considera un proceso cultural, tecnológico, político, social, etc. De esta forma, si una mayoría de noticias se encuentran en la sección de "Tecnología", la lectura será considerada en este período y por este medio de comunicación como un proceso meramente tecnológico, mientras que si estas se incluyen en la sección de "Cultura" se tratará de un concepto eminentemente cultural.

Para ello se tienen en cuenta las secciones que ocupan el conjunto de noticias sobre lectura en el periódico El País entre 2004 y 2010. Estas son las siguientes: en "Cultura" tenemos 5 noticias; en "Comunicación", 1; en "Vida y artes", 2; en "Opinión", 2; en "Sociedad", 2; y una en "Revista de Verano".

En primer lugar, tal y como ocurría en el período anterior, tenemos una mayoría de noticias en la sección de "Cultura", todas ellas relacionadas con los resultados de encuestas de índices lectores en nuestro país - La frecuencia de lectura y el número de lectores crecieron durante 2004 del 07/02/2005, Muchos libros, pero pocos lectores del 11/10/2007-, la industria editorial Enganchados al 'thriller' histórico: La mitad de los 30 libros más leídos en 2006 en España sigue la senda de 'El código Da Vinci' del 22/2/2007 y El libro de bolsillo inyecta vitalidad al sector editorial, según los datos de 2005 del 09/09/2006 - y sobre hábitos culturales — Los españoles prefieren el cine y la música entre las opciones culturales del 01/04/2005.

En "Opinión" encontramos un total de 2 artículos: Libros y lectores del 10/07/2005 y Cautivos $y$ desarmados ante las elecciones del $05 / 03 / 2008$. La primera sin firma y la segunda tiene como autor a Gonzalo Pontón. Ambas comentan los resultados obtenidos en los diferentes estudios de hábitos lectores, pero la se- gunda tiene además reflexiones de índole política.

Los textos que encontramos en "Sociedad" se centran en la situación en la que se encuentra la prensa en esos momentos: Los españoles compran más prensa hoy que hace cinco años del 19/08/2007 y Los editores de diarios lanzan un plan para atraer a los jóvenes del 12/12/2007. Las dos son de 2007, año en el que se temía por la desaparición de la prensa impresa debido al éxito que tenía la prensa digital. Se trataba de un fenómeno mundial, que afectaba a muchos países del mundo, por lo que las noticias reflexionaban acerca de las distintas medidas a llevar a cabo para que los editores de diarios pudieran salir a flote. Pero, si se habla de la tipografía de los periódicos, entonces el texto se inserta en la sección de "Comunicación": Prensa. Francia. 'Le Monde' reduce tamaño, aumenta el tipo de letra y da más fotografías del 7/11/2005.

En "Vida y artes" hasta ahora no habíamos encontrado noticias relacionadas con la lectura, sin embargo ahora tenemos dos: La cultura ya es de masas del 03/03/2009 y El futuro de la poesía no cabe en los libros del 12/08/2009; en las que se reflexiona sobre el papel que tiene la lectura como proceso cultural en la sociedad y vida diaria de los españoles.

Concluimos así, que a pesar de que sigue habiendo un grupo considerable de noticias en la sección "Cultura", el número es inferior al del período temporal anterior. Posiblemente se deba a que la influencia de las nuevas tecnologías esté haciendo mella en el proceso lector.

Por otro lado, a medida que avanzan los años encontramos una dispersión cada vez mayor de noticias en las diferentes secciones de los periódicos. Muestra de ello es, por ejemplo, el reducido número de textos que aparecen en "Opinión" frente a períodos anteriores, lo que probablemente se deba a los recortes que se están dando en los diferentes medios de comunicación por la crisis económica por la que está atravesando el país en esos años. Esto se debe a que los artículos de opinión firmados por autores relevantes son muy costosos, por lo que este tipo de textos son de los primeros de los que se prescinde.

Si a esto añadimos la proliferación de suplementos culturales y tecnológicos que se publicaron junto a los periódicos durante estos años, repararemos en que muchas de las noticias sobre lectura se empiezan a incluir en los mismos. Esto puede ser un inconveniente a la hora de hacer un seguimiento de las noticias sobre el tema por parte de los aficionados a la lectura, 
por lo que no contribuye al fomento de la misma.

\section{Conclusiones}

El análisis de las noticias sobre lectura publicas en El País ofrece conclusiones muy interesantes, especialmente si se contrastan con los resultados disponibles para periodos anteriores.

Algunos términos estaban presentes ya en la fase previamente estudiada. En primer lugar, aparece 'clasificación' de lectura, como se puede ver en el Gráfico II, concepto que encontrábamos también en el anterior rango temporal de este mismo periódico. Se refiere al tipo de géneros preferidos - representado en el gráfico con 'prefiere'- para la lectura. Además de describir los gustos literarios de los lectores, se hacen 'recomendacion[es]' de obras o libros específicos, animando así a la lectura de las mismas.

También se repite con respecto al período anterior el término 'información', referido a los diferentes informes y estudios acerca de los hábitos lectores.

Pero hay algunas novedades importantes. Así, aparece 'medida' con el que se insiste sobre el hecho de ofrecer cantidades aproximadas acerca de los hábitos de lectura, es decir las preferencias de los procesos de lectura de la mayoría, minoría, etc. También se documenta 'radio', con el que se explican las diferentes actividades que la población española realiza en su tiempo libre: leer, ver la televisión, escuchar la radio, etc. Esto resulta novedoso, ya que se trata de un medio de comunicación antiguo si lo comparamos con el desarrollo de los nuevos dispositivos relacionados con Internet y, sin embargo, este último no está presente. Esto es debido a que hay diversas palabras para designar cada medio de comunicación, pues sí que se mencionan los nuevos formatos de lectura a través de conceptos relacionados como lectura en 'papel', 'materialidad' y 'tacto', haciendo referencia a los libros impresos, en contraposición a los electrónicos. Esto es un avance con respecto a los períodos anteriores, ya que hasta el 2008 no empiezan a proliferar de forma significativa este tipo de formatos. Eso sí, se mencionan también otros medios como 'televisión' y 'periódico'.

Podríamos concluir que, durante este rango temporal y en este periódico en concreto, predomina una visión positiva de la lectura, ya que aparecen asociados a ella términos como 'placer' de la lectura y 'alegría', por lo que se habla de los beneficios que tiene la lectura en la vida de las personas. Se trata de algo diferente de lo que habíamos visto hasta ahora, ya que si ob- servamos los resultados obtenidos en este mismo periódico en el período del 1982 al 1996 encontramos 'aversión' a la lectura y en el período al que nos referimos ahora 2004-2010 en otros periódicos como El Mundo o $A B C$ encontramos 'necesidad' de lectura.

Eso sí, frente a nuestras expectativas iniciales, el impacto de las nuevas tecnologías aún no está presente de forma mayoritaria en el proceso lector. A pesar de que es durante este rango temporal cuando surgen los nuevos dispositivos de lectura, su uso no llega a ser mayoritario, manteniéndose el concepto de lectura como un proceso meramente cultural. Se vislumbra que en años posteriores pueda llegar a tener tintes más tecnológicos, por lo que esta podría ser una interesante línea de trabajo futuro.

Por otro lado, a pesar de que uno de nuestros objetivos era comprobar si los cambios de gobierno en España habían afectado al contenido de las noticias publicadas durante este período, a diferencia de los anteriores, podemos afirmar que no ha tenido lugar ningún cambio relevante. Es decir, aunque sí que encontramos alguna noticia con influencia política en las noticias con fechas próximas a las elecciones locales de 2007, no aparecen en un número destacable.

Tal y como adelantábamos en el apartado 5, con el fin de fomentar la lectura sería interesante que los medios de comunicación incluyeran las noticias sobre lectura en una misma sección del periódico para facilitar así un seguimiento de las mismas. Es más, el hecho de que los textos aparezcan en suplementos culturales o tecnológicos - y no en el periódico en sí- implica que, al tratarse de publicaciones especializadas, sean probablemente leídas únicamente por los usuarios aficionados a la lectura. De nuevo, esto no ayuda a fomentar la lectura.

Con el mismo objetivo, sería bueno que las convocatorias de premios al fomento de la lectura, tanto de instituciones como de autores, se incluyeran en la prensa. Hemos comprobado que estas aparecen en el Boletín Oficial del Estado con el fin de que puedan presentarse los candidatos; sin embargo, en la prensa tan solo aparecen noticias sobre los premiados y las ceremonias de entrega de los premios. Si se publicaran en los medios se daría mayor publicidad a los mismos, motivando así la promoción de la lectura.

\section{Referencias}

Achaerandio, Rafael (2010). Observatorio de piratería y hábitos de consumo de contenidos digitales: Primer semestre de 2010. Madrid: Cedro. http://www.cedro.org/ 
Files/Observatoriopirateriayhabitosconsumodigitales.pdf (10 de mayo de 2011).

Aguirre Romero, Joaquín María (2007). Apuntes sobre el cambio: entre dos mundos: las TIC, la cultura y los jóvenes. // Primeras Noticias. 225 (2007) 15-24.

AIMC (2010). Audiencia de Internet. Madrid: AIMC, 2010.

Almuiña, Celso; Sotillos, Eduardo (2002). Del periódico a la sociedad de información. Madrid: Sociedad Estatal España Nuevo Milenio, 2002. 3 v. 84-95486-50-4 (o.c.).

Armentía, José Ignacio, [et. al.] (2000). El diario digital y las nuevas fórmulas de lectura. // Trípodos. Extra (2000) 455-473.

Asociación para la investigación de medios de comunicación (2010). Navegantes en la red. Madrid: AIMC, 2010.

Bahloul, Joëlle. (2002). Lecturas precarias: estudio sociológico sobre los "pocos lectores". México, D. F: Fondo de Cultura Económica.

Barómetro de hábitos de lectura y compra de libros en 2007. (2007). Madrid: Federación del Gremio de editores de España, 2007. http://www.ediciona.com/documents /recursos/barometro_habitos_lectura_y_compra_de_libr os_espana_2007.pdf (10 de mayo de 2011).

Barómetro de hábitos de lectura y compra de libros en 2008. (2008). Madrid: Federación del Gremio de editores de España, 2008. http://www.federacioneditores.org/0_Re sources/Documentos/Indice_Lectura_Esp_2008.pdf (10 de mayo de 2011)

Barómetro de hábitos de lectura y compra de libros en 2009. (2009). Madrid: Federación del Gremio de editores de España, 2009. http://www.federacioneditores.org/0_Res ources/Documentos/NP Lectura_TercerTrimestre 2009 .pdf (10 de mayo de 2011).

Barómetro de hábitos de lectura y compra de libros en 2010. (2010). Madrid: Federación del Gremio de editores de España, 2010. http://www.federacioneditores.org/0 Re
sources/Documentos/NP Lectura 2Cuatrimestre.pdf (10 de mayo de 2011).

Chartier, Roger (2000). Las revoluciones de la cultura escrita: diálogo e intervenciones. Barcelona: Gedisa, 2000a. 84-7432-829-2.

Cordón García, José Antonio. Los libros electrónicos: una realidad emergente. // Anuario ThinkEPI. 4 (2010) 130138.

Cordón García, José Antonio (2011). El fin del libro y el principio de la lectura: los libros electrónicos y el fenómeno Ipad. // Anuario ThinkEpi. 5 (2011).

Cordón García, José Antonio; Alonso Arévalo, Julio (2010). Los libros electrónicos: nuevas formas de edición y nuevos modos de lectura. Madrid: Une Libros, 2010. 21-23.

Cordón García, José Antonio; Alonso Arévalo, Julio; Martín Rodero, Helena (2010b). Los libros electrónicos: la tercera ola de la revolución digital. // Anales de Documentación. 13 (2010) 53-80.

Cordón García, José Antonio, Gómez, Raquel; Alonso Arévalo, Julio (2011). Gutenberg 2.0. Madrid: Trea, 2011d.

García-Delgado Giménez, Belén (2012). Estudio de la lectura en los discursos legislativos y medios de comunicación social. Salamanca: Ediciones de la Universidad de Salamanca, 2012. 987-84-9012-135-1.

Lahire, Bernard (2004). Sociología de la lectura. Barcelona: Gedisa, 2004. 84-9784-020-8.

Millán, José Antonio (2008). La lectura en España. Informe 2008. Leer para aprender. Madrid: Fundación Germán Sánchez Ruipérez, Federación de Gremios de Editores de España, 2008.

Enviado: 2012-07-28. Segunda versión: 2013-0-. Aceptado: 2013-0-. 
